\title{
空间增强现实中的人机交互技术综述
}

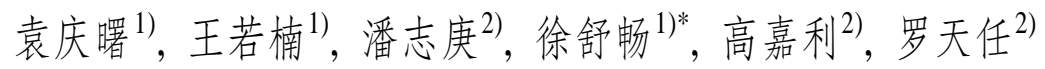 \\ 1) (杭州师范大学信息科学与工程学院 杭州 311121) \\ 2) (杭州师范大学阿里巴巴商学院＼cjkstart杭州 311121) \\ (xusc@hznu.edu.cn)
}

\begin{abstract}
摘 要: 空间增强现实是增强现实的三大形式之一, 它利用投影等方式在物理空间中增强显示虚拟内容. 在空间增 强现实系统中，用户可以在物理空间与虚拟内容交互，具有良好的环境感知、空间感知与协同感知，交互任务执行过 程中认知负荷低. 首先以空间增强现实显示方式为例，通过其系统架构介绍空间增强现实的技术原理；然后将空间 增强现实中的人机交互技术分类为基于触控的交互、基于手势或姿势的交互、基于控制器的交互、手持投影仪交互、 实物交互及多模态交互并进行分析，对相关技术在三维配准、虚实融合等方面带来的挑战进行阐述; 同时总结了空间 增强现实在运动锻炼、医疗辅助、教育娱乐、艺术等领域的应用状况; 最后对空间增强现实中的交互技术发展趋势 进行了展望，指出未来要克服现有技术在稳定性、准确性和实时性上存在的缺陷，在交互方式更自然、交互遮挡处理 方法更智能、系统使用场景更广泛、与社会文化和心理现象研究相结合以及新型成像方式等方面得到突破和发展.
\end{abstract}

关键词：增强现实；空间增强现实；投影映射；人机交互；人机界面；实物交互

中图法分类号: TP391.41 DOI: $10.3724 /$ SP.J.1089.2021.18445

\section{A Survey on Human-Computer Interaction in Spatial Augmented Reality}

\author{
Yuan Qingshu ${ }^{1)}$, Wang Ruonan ${ }^{1)}$, Pan Zhigeng ${ }^{2)}$, Xu Shuchang ${ }^{1{ }^{*}}$, Gao Jiali ${ }^{2)}$, and Luo Tianren ${ }^{2)}$ \\ 1) (School of Information Science and Engineering, Hangzhou Normal University, Hangzhou 311121) \\ 2) (Alibaba Business School, Hangzhou Normal University, Hangzhou 311121)
}

\begin{abstract}
Spatial augmented reality (SAR), which typically uses projectors to augment physical spaces with virtual contents, is one of the three main forms of augmented reality. In SAR systems, users can interact with virtual contents in physical spaces, therefore they have good environmental, spatial and collaborative perceptions. SAR systems can also reduce the cognitive load of interaction tasks. SAR display forms are firstly demonstrated, and then SAR architectures are illustrated as well as its technical principles. SAR interaction technologies are classified into 6 categories, which are touch based interaction, gesture or posture based interaction, 3D controller based interaction, handholding projector interaction, tangible interaction, and multimodal interaction. Challenges from 3D registration, virtual-real fusion are analyzed. The application status in the field of sports, medical treatment, education, entertainment and art are discussed. The trends in SAR interaction are finally predicted. Specifically, SAR interaction will become more natural, more intelligent in occlusion processing, more widely applicable, more closely related with the social, cultural and psychological researches, and novel display approaches will also be used by SAR systems.
\end{abstract}

收稿日期：2020-06-22; 修回日期：2020-12-09. 基金项目：国家重点研发计划(2018YFB1004901); 浙江省自然科学基金 (LY19F020020); 教育部产学合作协同育人项目(201902227001). 袁庆曙(1979一), 男, 博士, 讲师, CCF 会员, 主要研究方向为增强现 实、人机交互; 王若楠(1996-), 女, 硕士研究生, 主要研究方向为增强现实、人机交互; 潘志庚(1965一), 男, 博士, 教授, 博士生导 师, CCF 会员, 主要研究方向为虚拟现实、人机交互; 徐舒畅(1979-), 男, 博士, 副教授, 硕士生导师, 论文通讯作者, 主要研究方向 为图像处理、计算机视觉、模式识别与人工智能; 高嘉利(1996一), 女, 硕士研究生, 主要研究方向为增强现实、人机交互; 罗天任 (1995一), 男, 硕士研究生, 主要研究方向为增强现实、人机交互. 
Key words: augmented reality; spatial augmented reality; projection mapping; human-computer interaction; hu-

man-computer interface; tangible interaction

增强现实是一种将虚拟信息自然地融合到真 实世界中的技术，根据虚拟信息呈现位置的不同可 以分为头戴式、手持式和空间式 3 大类. 其中, 空 间增强现实(spatial augmented reality, SAR)是一种 借助各种设备, 以物理空间作为显示介质呈现虚拟 物体的像达到虚实融合效果的技术 ${ }^{[1]}$. SAR 将显示 设备与用户分离, 减少了用户操作时的交互负载和 认知负荷; 显示内容不与具体用户个体相关联, 允 许多个用户从不同的方位观察虚实融合显示的场 景，具有良好的协同感知; 用户在物理空间中执行 交互任务，具有良好的环境感知和空间感知.

SAR 的起源可以追溯到 20 世纪 60 年代，当时 被称作投影映射或着色灯技术. 1969 年，迪士尼公 司 ${ }^{[2]}$ 将提前录制下来的歌手头部影像投影到他们 的半身雕像上, 让这些雕像看起来像是在歌唱的 真人, 如图 1 所示. 1980 年, 装置艺术家 Michael Naimark 录制了一段人们与房间中物体互动的影 像，并把它投影在房间内，创造出人们还在房间中 的错觉 ${ }^{[3]}$. 直到 1990 年末, 北卡罗来纳大学教堂 山分校的研究者 ${ }^{[4]}$ 利用投影把不同地点的活动显 示在同一个投影环境中，投影映射这个概念才正 式在学术界提出来，该项目被称为“未来办公室”. 之后，投影映射技术蓬勃发展，越来越多的艺术家 将其使用在他们的作品中, 也有更多的科学家和工 程师将前沿的技术整合其中, 以拓宽其应用场景.

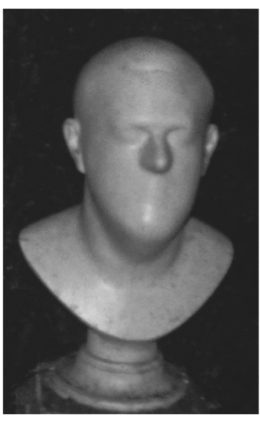

a. 原始雕像

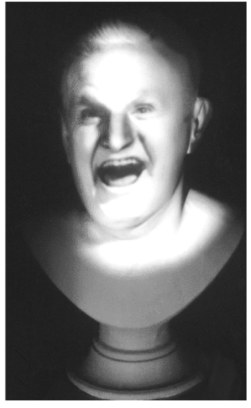

b. 投影映射后的雕像
图 1 迪士尼公司幽灵鬼屋项目的歌唱雕像 ${ }^{[2]}$

人机交互是 SAR 的重要支撑技术，正向着自 然化、智能化、高效化及实体化方向发展. 新兴交 互技术的出现很大程度上促进了 SAR 交互系统和 应用的发展. 然而, SAR 中的人机交互技术在交互 界面、交互范式和交互技术等方面还面临着一些挑 战性问题。

\section{SAR 技术原理}

SAR 在物理空间中的显示方式, 主要有基于 显示屏的视频穿透显示、空间光学穿透显示和基于 投影的空间显示 ${ }^{[5]} 3$ 种. 基于显示屏的视频穿透显 示的原理是, 将现实图像通过相机实时采集并合 成虚拟图像, 最终在显示屏上显示, 如图 2a 所示. 空间光学穿透显示的原理是, 将来自现实环境的 透射图像和虚拟环境的反射图像合成于光学合成 镜片中, 平面或弯曲反射光束合路器或光学全息 图等是此类显示器的基本组件, 如图 $2 \mathrm{~b}$ 所示. 基 于投影的空间显示方式，利用投影仪把虚拟图像 信息直接投影到现实场景中的对象表面，提供高 分辨率和可扩展的分辨率、大范围和可扩展的视 野. 此外, 眼睛更容易调节适应度, 防止由于瞳距 不匹配而导致的视差失真 ${ }^{[6]}$, 许多研究项目都强调 了投影仪在现实世界之上模拟新颖、友好界面的好 处 ${ }^{[7-8]}$, 如图 2c 所示. 基于投影的 SAR 的缺点是投 影光路可能会被遮挡，对光照环境和投射材质有 一定的要求, 另外其安装部署也相对较难.

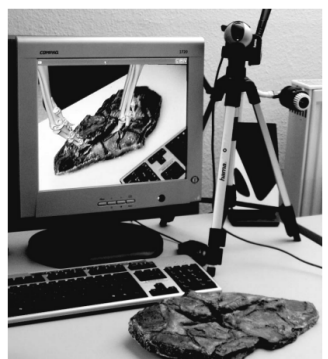

a. 基于显示屏的 视频穿透显示 ${ }^{[2]}$

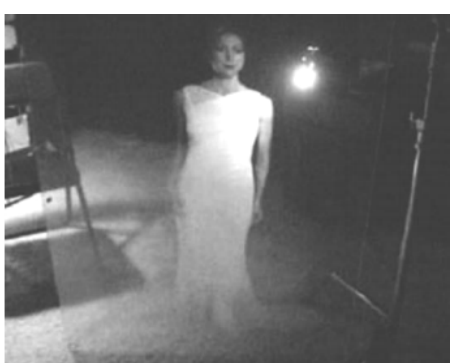

b. 空间光学穿透显示 ${ }^{[2]}$

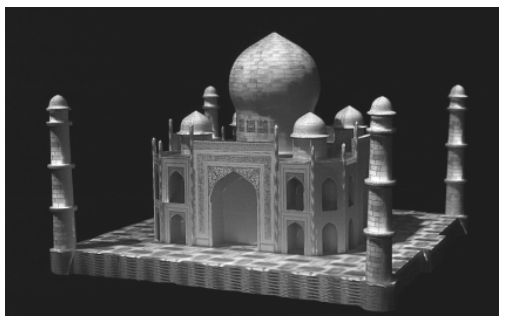

c. 基于投影的空间显示 ${ }^{[3]}$

图 2 SAR 显示应用示例

图 3 所示为 SAR 系统架构, 其中在成像原理 上，视频穿透显示、空间光学穿透显示和基于投影 的空间显示分别如 (a), (b), (c) 所示. 


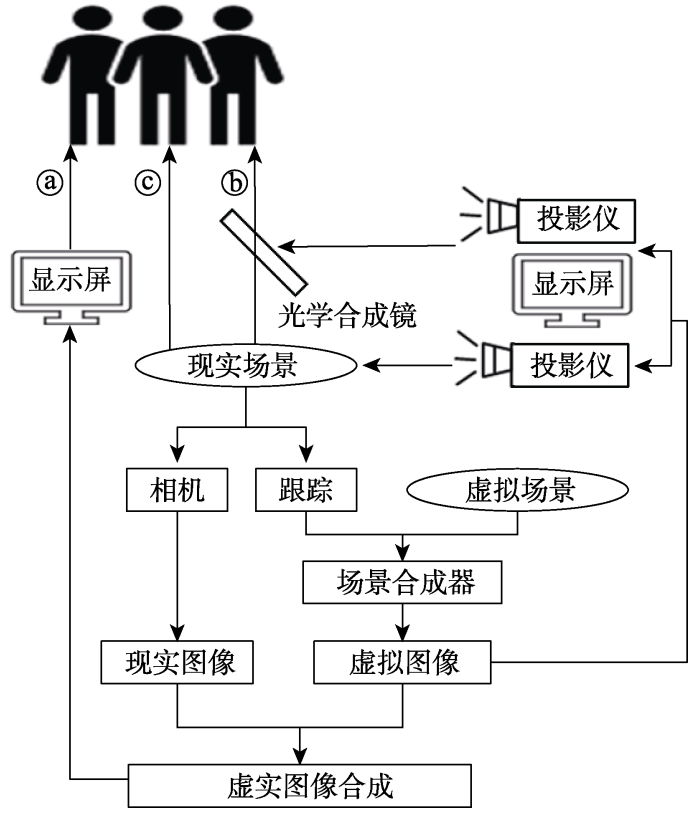

图 3 SAR 系统架构

\section{SAR 中的人机交互技术分类}

增强现实的研究最初主要集中在跟踪、注册和 显示上, 强调将虚拟物体叠加在真实场景上, 如头 戴式和手持式显示器或空间投影的可视化 ${ }^{[2,9]}$. 随 着技术的发展，用户对增强现实中的交互需求变 得更为强烈，促使了多种交互技术的发展. 如图 4 所示, SAR 交互技术主要可以分为：基于触控的交 互、基于手势或姿势的交互、基于控制器的交互、 手持投影仪交互、实物交互和多模态交互.

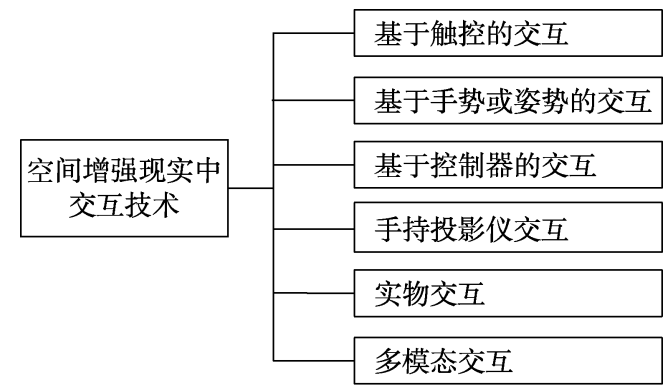

图 4 SAR 中交互技术分类

\section{1 基于触控的交互}

基于触控的交互主要是通过获取手指触摸的 位置信息，触发相应命令，完成对虚拟物体的选 择、移动和旋转等操作. 它是一种基于图形用户界 面 (graphical user interface, GUI), 以触觉作为主要 感知通道的交互技术.

Duopography 系统 ${ }^{[10]}$ 能对物理地形模型进行
投影显示, 同时支持多点触控功能进行地形的缩 放. 通过对手指位置的检测, 用户可实现平移、缩 放和旋转等功能, 还可以使用手指直接在模型上 草绘. OmniTouch ${ }^{[11]}$ 是可穿戴的投影系统，可在对 象表面上实现交互式多点触摸应用; 它使用深度感 知实现多点触控手指跟踪, 无需进行校准. Hochreiter 等 ${ }^{[12]}$ 通过检测和定位手指触控事件, 并发送到 渲染系统, 投影出适当的图形模型. Dung 等 ${ }^{[13]}$ 提 出了一种基于视觉的触控技术, 利用手指与其阴 影的相对关系检测触控事件和位置，用于交互式 投影.

触控交互以其自然直观的操作方式成为 SAR 重要的交互手段之一, 最大的优点是与图形用户 界面兼容或相似，便于用户理解和操作，摆脱了鼠 标和键盘等设备的限制. 这种方式是对 WIMP (window, icon, menu, pointer)界面范式的扩充, 即 用触控代替光标的指点, 交互的对象通常是数字 空间中的纯数字内容.

\section{2 基于手势或姿势的交互}

基于手势或姿势的交互利用人的自然手势与 身体姿态作为交互输人通道, 通过视觉中目标跟 踪方法获得人在物理空间的手势或姿势信息, 并 将其作为 SAR 系统的输人, 用于实现虚拟对象的 选择与控制.

Wilson 等 ${ }^{[14]}$ 对多个深度相机和投影仪标定, 实现在任何表面上精确地投影图案，可通过手势 识别实现与投影内容的交互. 陈锐等 ${ }^{[15]}$ 基于多个 摄像头对任意方向的人脸朝向进行判断, 驱动云 台根据朝向带动投影仪旋转, 将投影方向调整到 与人脸朝向一致的方向, 实现了投影仪投影方向自 动适应人脸朝向的人机交互方式. 第六感(SixSense) 装置 ${ }^{[16]}$ 能让对象表面或物体变成交互式的显示媒 介，用户通过手势即可与虚拟信息进行互动，如用 户可以用手指比划出一个方框完成一幅摄影作品. 此外，还有一些接触式的手势或姿势交互技术，主 要利用数据手套、智能手环、数据衣以及肌电检测 等实现交互, 由于需要穿戴设备, 舒适性较差, 因 此在 SAR 中的应用并不多见.

手势和姿势是现实生活中人与人交流及信息 交换的主要方式, 被 SAR 系统所采用, 体现了增 强现实的“现实感”, 更体现了计算机的“拟人化”趋 势. 与基于触控的交互相比, 手势交互行为通常不 需要与虚拟内容直接接触即可触发相应的命令; 而触控行为需要发生直接接触, 基于触控的交互 技术本质上还是一种围绕桌面隐喻展开的交互形 
式. 基于手势或姿势的交互最大的优势在于自然, 即与日常生活贴近. 手势交互语义应该符合日常 人手操作物体的交互行为习惯 ${ }^{[17]}$. 由于手势本身 的多义性、人手形态变化的高维度以及手指之间存 在无法预测的遮挡, 手势的识别是一项具有挑战 性的研究. 它的困难在于难以区分连续运动过程 中用户的哪些动作是有意识的交互行为, 哪些动 作是下意识的动作. 在连续、精准地控制对象方面, 手势与姿势的交互稳定性还有待提高. 此外，当一 个系统使用过多的手势或姿势交互时，会给用户 带来记忆和认知的负担.

\section{3 基于控制器的交互}

基于控制器的交互主要利用光投射器、空间位 置跟踪器、交互笔或手柄等控制设备进行交互, 将 控制器的 6 自由度位姿、按钮状态和加速度等信息 赋予或重定向增强现实中的虚拟对象上，实现对 象的选择与控制.

Bandyopadhyay 等 ${ }^{[18]}$ 通过把光学跟踪器连接 到真实对象上, 把电磁跟踪器连接到笔刷上, 预先 获取对象的几何和纹理坐标, 并把投影仪校准到 跟踪器的参考系, 实现可移动真实对象的三维空 间增强绘画和投影. 陈锋等 ${ }^{[19]}$ 提出了一种基于多 通道投影系统的纹理表面实时绘制技术，用户通 过在平板交互设备上进行绘制，可以实现对投射 在物体表面图案的实时修改. Roo 等 ${ }^{[20]}$ 提出了一个 由 SAR 和虚拟现实组成的混合空间，用户通过按 下手柄控制器的触控板切换视野，完成虚拟空间 到现实空间的转换; 同时可使用手柄控制器实现 抓取、改变虚拟对象的位置方向，或在目标对象上 绘制标注.

基于控制器的交互能很好地将用户的运动赋 予或重定向到虚拟对象上. 增强现实中基于控制 器的移动可以放大或缩小用户的实际移动距离, 十分灵活. 基于控制器的交互已经在游戏、虚拟驾 驶等方面得到了广泛的应用. 但由于这种交互方 式需要将控制器的运动赋予虚拟对象, 其中重定 向的过程使虚拟对象的运动与控制器的运动处于 相对分离状态，因此用户在使用时需要一定的熟 悉与学习过程, 才能适应这种交互方式.

\section{4 手持投影仪交互}

手持投影仪交互是一种 SAR 的特殊交互形式, 它将 SAR 的交互输人与显示输出相结合, 即用户 改变作为显示设备的投影仪的姿态，结合位置跟 踪器、投影仪上的按钮和激光笔等, 动态地调整投 影显示的内容.
$\mathrm{Cao}$ 等 ${ }^{[21-22]}$ 提出了一套交互技术，用户手持投 影仪在墙壁上投射出图像，同时配合光学三维跟 踪的触控笔, 实现画面的切换和按钮的点击等功 能，该系统还支持多人手持协作投影同一画面. Santos 等 ${ }^{[23]}$ 介绍了鬼屋，使用相机捕获房间中玩 家的身份、位置和行为, 玩家可以在手持投影仪前 比划手势与呈现出的虚拟角色互动, 如玩家在空 中画圆圈时, 可以听到令人恐惧的声音. Willis 等 ${ }^{[24]}$ 设计并实现了 SideBySide 系统, 通过使用相机和 红外光投影仪跟踪不可见基准标记，进而跟踪多 个投影内容, 多用户可手持投影设备进行游戏和 互动.

手持投影仪具有结构紧凑、可便携、功耗低和 待机长等特点, 其最大优点就是便携性, 为 SAR 的交互提供了一条有效的途径. 但手持投影仪使 用户的手被占用，这给用户交互的自然性和舒适 感带来了一定的挑战.

\section{5 基于实物的交互}

Ullmer 等 ${ }^{[25]}$ 提出实物用户界面(tangible user interface, TUI)的概念, 即把数字信息连接到日常 物理对象或环境中, 以增强物理世界, 使数字信息 可以直接通过用户的双手进行操纵，如图 5 所示. 这种交互形式称为“空间增强现实实物交互”, 比 较适用于 SAR 系统, 其最核心的理念是将可触的 实物输人与不可触的数字呈现在物理空间上统一 起来.

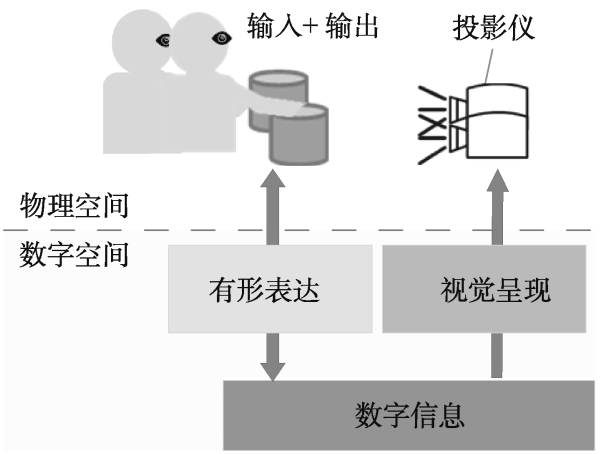

图 5 TUI 实物操作界面

SAR 中的实物交互技术根据实物本身的作用 不同，可以分为以下 3 大类.

(1) 将 TUI 技术与二维图形用户界面相结合, 实物对象作为图形用户界面的触发装置或图形显 示单元. Jones 等 ${ }^{[26]}$ 通过识别多个实物对象上的标 签, 将标签的运动赋予一定的语义, 实现交互桌面 上显示内容的缩放、激活和切换等操作，如图 6 所 示, 其中框内的为实物对象. de Raffaele 等 ${ }^{[27]}$ 利用 
桌下的相机识别交互桌面上实物交互对象底面的 标签(交互桌面可透光), 从而实现控制输人, 并利 用投影仪改变交互桌面的数字内容. Attard 等 ${ }^{[28]}$ 使 用相机识别白板上实物对象的位置与方向，提供 了白板上的实物交互功能, 类似的工作还有文献 [29]等. 该类方法多见于实物交互桌面，它将实物 形式与二维图形用户界面相结合, 交互行为发生 在二维空间中. 由于 GUI通常被用户所熟知，因此 这种交互方式很容易被用户接受与理解，它本质 上是对 WIMP 范式的改进, 利用了人们熟悉的桌 面交互隐喻. 它的不足之处是, 虽然用到了实物的 形式，但没有充分发挥实物交互天然具备的空间 隐喻优势.

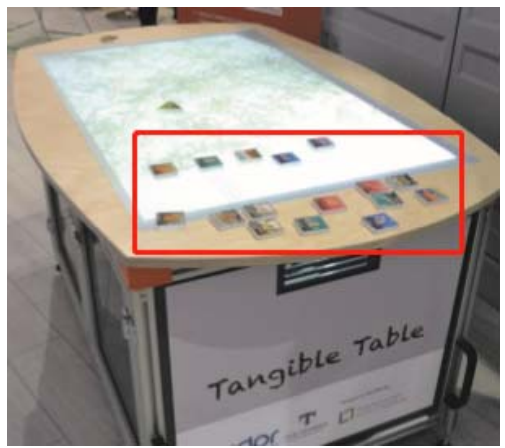

图 6 实物交互桌面 ${ }^{[26]}$

(2) 将 TUI 技术与三维图形用户界面或三维 空间相结合. Sano 等 ${ }^{[30]}$ 提出的 Rubix 系统, 利用 Kinect实时输出的点云检测多个平面，并检测空间 中的立方体对象, 能够提供立方体对象的 6 自由度 跟踪数据; 通过对每个平面提供纹理映射, 让用户 在物理空间看到三维图形对象. Gervais 等 ${ }^{[31]}$ 在实 物对象和屏幕之间创建映射关系，可以将实物三 维空间中的交互事件映射到二维图形空间中，同 时允许二维屏幕上的光标以无缝的方式移动到实 物对象上. Oswald 等 ${ }^{[32]}$ 介绍了 i.Ge引擎，通过在物 理空间中识别不同颜色的标签，定义了每个标签 的交互语义, 从而改变游戏内容，如关卡信息、角 色信息等, 如图 7 所示. 这类研究突破了二维 GUI 的限制, 交互行为在三维空间中展开, 充分利用了 空间隐喻, 实物在交互中所起的作用与第(1)类基 本类似.

（3）实物对象的交互式投影上色, $\mathrm{Pmomo}^{[33]}$ 采 用 Kinect 深度相机实时捕获场景并计算平移矢量, 用 AHRS 传感器获取旋转矢量, 基于 CMA-ES 方 法跟踪实物对象的 6 自由度，并通过投影虚拟内容 对单个实物对象进行着色. Kurth 等 ${ }^{[34}$ 采用结构光

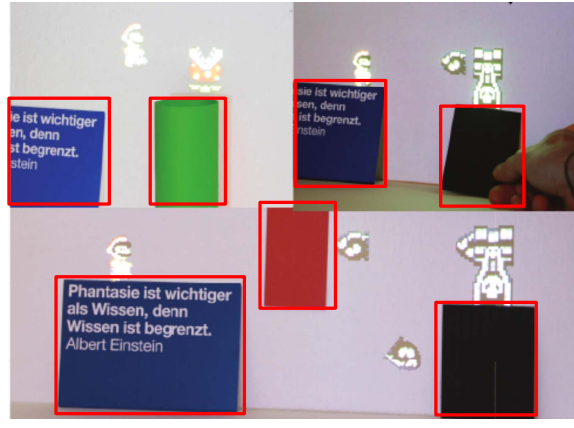

图 7 i.Ge 引擎 ${ }^{[32]}$ (框内为交互用的实物对象)

确定相机投影的点对应关系来重建场景点云, 将 其与深度相机检测的对象点云匹配, 以求解 RGB 相机与深度相机的旋转平移信息，迭代更新相机、 投影仪的外部参数, 最终用于单个对象的动态投 影着色, 如图 8 所示. Kagami 等 ${ }^{[35]}$ 通过在投影内容 中嵌人黑白的特征图案，循环迭代得到对象的跟 踪参数, 实现投影内容的精确着色. Punpongsanon 等 ${ }^{[36]}$ 通过投影增强织物运动外观, 允许用户操纵 观察特定应用场景下织物的弯曲刚度. Lumipen2 系统 ${ }^{[37]}$ 采用高速跟踪技术通过镜射照明观察目标 物体的轮廓, 实现了对包括投影图像在内的光照 变化不加任何标记的单个简单几何体进行鲁棒高 速跟踪. 这类系统也叫投影映射, 其目标是对实物 对象进行精确的投影着色. 近年来, 这类系统以移 动实物对象的快速动态投影上色为研究目标, 通 常使用如图 9 所示的流程实现, 即通过计算机视觉 等方法实时检测对象的位姿，利用相机、对象及投 影仪的相对关系约束计算投影图像，完成交互式 上色. 如何快速、精确地求解多坐标之间的相对关 系，是这类技术的核心问题所在. 这类交互的优点 是充分将物理空间的实物与数字空间的内容结合 于实物交互对象上，实现真实世界与虚拟世界的

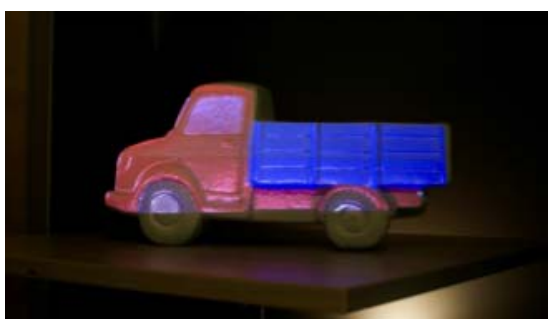

图 8 实物对象交互式投影上色 ${ }^{[34]}$

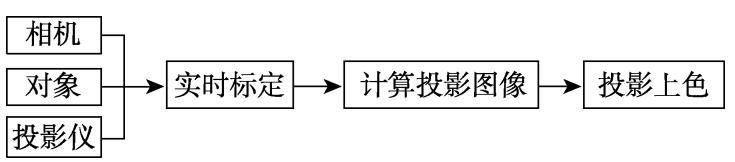

图 9 实物对象投影上色实现流程 
无缝融合. 由于系统的延迟和精度等问题, 有时会 带来投影着色的不同步，进而影响交互体验.

实物交互将物理形式赋予数字信息，让其表 示和控制对应的数字信息, 使数字信息可以直接 被用户的双手操纵, 充分运用了人类在与现实世 界进行交互时所依赖的自然运动技能，使用户在 一个互动环境中实现环境中自由运动并与实物场 景进行交互. 实物交互支持时分复用和空间复用, 同时可实现用户协同操作. 由于实物交互充分利 用了用户熟悉的现实环境，因此实物交互的认识 负荷低，满足了用户自然交互的需求。但在 TUI 中, 虚拟三维对象只能叠加于实物对象表面上, 而 不是在三维空间的任意位置上，因此，交互被限制 在对象表面上. 此外, 实物交互过程中实物对象通 常难以进行非刚性形变.

\section{6 多模态交互}

不同形式的输人组合(如语音、手势、触摸、 文字等)称为多模态交互，其目标是向用户提供与 计算机进行交互的多种选择方式，以支持自然的 用户选择，在这种交互方式中通常需要维持不同 模态间的一致性 ${ }^{[38]}$.

SAR 环境中的许多应用都利用了多模态交互 技术. 例如, Benko 等 ${ }^{\left[{ }^{39]}\right.}$ 提出的 Mano-a-Mano 结合 了动态投影映射、多视角和无设备交互，通过碰撞 检测、语音或触摸等方式支持与三维虚拟对象的双 向交互. WUW 系统 ${ }^{[40]}$ 通过将虚拟信息投影到物体 表面、墙壁和周围的物理物体上，让用户通过自然 手势、手臂动作或物理物体的直接操控等多种途径 与投影的虚拟信息进行交互.

多模态交互的使用以并行的方式与计算机系 统进行交互，发挥了各个模态独特的优势，利用模 态之间的互补，带来了交互的自然性和高效性; 在 部分通道存在干扰时，多模态交互发挥了很大的 优势，提高了交互效率. 然而，相同的交互行为在 不同模态上下文中可能有不同的含义, 这使系统 难以给出准确的反馈. 因此, 不同模态之间交互信 息的融合和交互模态之间的管理，是多模态交互 的核心问题. 例如, 研究表明 ${ }^{[41]}$, 在一个语音和手 势结合的多模态系统中, 语音识别率和该语言是 否为该用户的母语状态显著相关，同时，手势识别 率也会随之改变.

\section{SAR 交互面临的挑战}

SAR 产生之初, 场景、对象与设备相对静止,
虚实配准的参数只需一次设置, 交互的过程中基 本不需要更改. 由于交互技术的发展, 给 SAR 在 三维配准、虚实融合等方面带来了新的挑战.

\section{1 交互行为容易引起视觉遮挡}

SAR 系统利用相机检测现实场景, 利用投影 仪在现实场景中投影虚拟图像. 交互的引人，使复 杂交互任务中人及交互对象会对 SAR 系统的投影 仪和相机的成像光路形成遮挡.

对于相机的光路被遮挡的问题，可以利用多 个相机同步检测来缓解，提前标定多相机之间的 外部参数, 在某个相机被遮挡时, 利用其他相机的 检测数据来代替 ${ }^{[42]}$.

对于投影仪的引起的光路遮挡问题，可以使 用多个不同机位的投影仪来解决, 即在某台投影 仪的光路被遮挡时，用其他投影仪来投影虚拟内 容. 由于多个投影仪投影出各自的画面，在重叠 区域会产生画面亮度的午余、投影仪强度和颜色 响应的非线性变化, 也会在接合处产生接缝, 这 就需要进行多投影的光度补偿和投影拼接等. 针 对该问题已经出现了多种解决方法, 如基于迭代 思想的多投影的光度补偿方法 ${ }^{[43]}$, 统一解决了在 复杂表面上的光度补偿和投影拼接问题, 并可将 其用于动态环境光的补偿. 此外, 使用 alpha 遮罩 也可改善重叠区域的过渡效果 ${ }^{[44-45]}$. 但由于现实 场景中投影表面的几何形状千变万化, 材质属性 也多种多样，给多投影画面的融合显示带来了一 定的挑战

\section{2 对象跟踪难以满足三维配准要求}

三维配准是任何增强现实系统所必须解决的 关键问题. 在 SAR 中, 需要利用计算机视觉等方 法确定对象相对于空间的位姿, 以便将虚拟场景 正确地注册到它应所处的位置上, 确定相对位姿 的过程通常称为对象跟踪 ${ }^{[46]}$. SAR 场景中对象跟 踪的主要困难在于场景的投影光对获取图像的影 响. 另外, 交互过程中目标对象的快速移动, 也会 加大对象跟踪的难度. 对象跟踪的精度、实时性及 更新率难以满足虚拟内容和真实世界对象注册的 要求, 是 SAR 面临的又一挑战.

在交互过程中, 当目标快速移动并变形时, 图 像处理的延迟和投影仪的延迟会导致目标物体与 投影图像之间的配准错误. 例如, 交互数据可能由 于对象遮挡、对象拍摄视角及照明条件的变化等原 因变得不稳定.

主流的虚实配准方法主要分为基于硬件设备 的和基于视觉的跟踪配准 2 大类. 基于硬件设备的 
跟踪配准技术通常借助电磁、超声、惯性、光学或 机械等跟踪器. 目前采用的跟踪器主要包括磁跟 踪器、螺旋测度仪、测距仪、超声波定位仪和全球 定位系统(global positioning system, GPS)等，用以 探测和跟踪真实环境中目标的位置和方向 ${ }^{[47]}$, 实 现增强的数字信息的实时注册. 跟踪器都有各自 的优缺点. 例如, 磁传感器具有较高的更新率并且 重量轻, 但是它们可能会受到附近的金属物质的 干扰；超声波定位仪易受环境噪声的影响; GPS 的 测量精度低，只适用于户外场景的位置移动应用 等. 文献[48]对基于传感器的跟踪进行了很好的回 顾. 此外，由于基于硬件设备的跟踪配准需要在交 互时制定一定的硬件装置, 交互过程中会给用户 带来一定的交互负载.

基于视觉的跟踪配准通常是利用计算机视觉 技术跟踪真实场景中的对象，并以此恢复场景中 对象的位姿, 最终计算参数进行虚实配准. 它是近 年来常用的 SAR 配准手段，其根据技术手段不同， 又可以分为基于自然特征的配准、基于标记的配准 以及基于深度数据的配准. 基于自然特征的配准 方法通常使用特征描述符, 如 SIFT(scale invariant feature transform) ${ }^{[49]}$ 算法通过检测目标图像中的自 然局部特征跟踪目标对象，但是该方法对动态场 景中的投影内容或照明条件敏感. 一种比较普遍 的做法是在相机上安装红外滤镜, 并配合照明场 景的红外灯，这样可以过滤投影仪投射的图案，从 而增加图像检测的稳定性. 一些系统通过在目标 对象上贴标记进行跟踪和定位, 如 MoSART 系统 ${ }^{[50]}$ 和 TangiBoard 系统 ${ }^{[28]}$. 它们有时会受到使用范围 有限和标记被遮挡导致跟踪错误的困扰，因为它 们仅在标记可见时才提供位置信息. 此外, 若系统 需要对多个对象进行跟踪, 将需要大量的标记, 因 此基于标记的方法扩展性差. 基于深度数据的配 准方法主要是基于深度图中的几何结构信息重建 对象的位姿，并进行虚实配准. 徐维鹏等 ${ }^{[51]}$ 使用 基于迭代最近点(iterative closest point, ICP)算法的 位姿估计和基于 SDF 的点云融合算法在线跟踪交 互场景, 得到实时的投影物体的位姿矩阵, 并生成 虚实物体的投影映射. 由于目前市场上深度相机 的帧率受到一定的限制，与使用二维信息跟踪配 准相比，使用深度相机实现低延迟的感官反馈一 直是一项具有挑战性的研究.

\section{3 缺乏普适的交互范式与交互界面支撑}

虽然近年来 SAR 中的交互技术发展迅速，但 每种交互技术都有一定的局限，如基于手势或姿
势的交互需要特定的手势或姿势, 不具有通用性, 需要用户的记忆, 且难以区分用户的哪些动作是 有意识的交互行为, 哪些是下意识的动作. 基于控 制器的交互需要借助第 3 方设备, 是一种间接的交 互方式, 需要一定的熟悉、适应及学习过程. 实物 交互方式对场景中多个复杂对象的同时交互支持 并不是很好.

优秀的交互界面需要可靠的交互范式指导, 正如桌面环境中的 WIMP 范式成就了二维 GUI. SAR 环境中, 用户与虚拟环境交互的输人和输出 是在三维空间中进行的, 这使得二维 GUI 组件已 经不能满足增强现实中的人机交互需求. 因此, SAR 中的人机交互也亟需研究具有普适指导意义的 交互范式, 考虑新的输人组件、输出显示和交互隐 喻, 在此基础上, 研究相对通用的交互界面及技术.

\section{4 使用场景受限}

相比手持与头戴式增强现实, SAR 系统支持可 扩展的视野及多用户的协同观察和操作，同时减 少了头戴式显示器的眩晕感, 并有可能集成到日 常的工作环境中 ${ }^{[52]}$. 另外，上述特性也使用户的 个人隐私信息难以得到保护, 因此 SAR 系统更适 用于公共空间，而不适用于个人空间。

SAR 系统使用前通常需要经过烦琐的校准步 骤, 且投影式 SAR 应用通常由多个投影仪组成, 这导致了系统使用空间庞大, 缺乏可移动性. 很多 系统仅停留在实验室阶段, 难以在实际场景中普 遍应用，这些都限制了 SAR 的使用场景.

基于投影的 SAR 系统由于需要在对象表面上 投影成像，因此在暗色吸光的表面或有高光反射 的表面上效果不佳. 此外, 由于容易受到环境光的 影响, 也较难在户外使用. 虽然利用高流明度激光 投影仪可以缓解该问题，但这仍然是制约其应用 场景的重要因素.

\section{SAR 的交互应用}

SAR 技术近年来在多个领域得到了广泛应用, 本文将从比较有代表性的运动锻炼、医疗辅助、教 育娱乐、艺术等领域对其应用状况进行综述.

\section{1 运动锻炼}

SAR 直接在物理空间呈现虚拟现象, 交互负 载小, 因此在运动锻炼方面, 它有着天然的优势. 研究表明, SAR 适合作为与身体活动结合的技术 ${ }^{[53]}$. 在运动锻炼中，一般利用 SAR 技术增强物理空间， 促进用户的行为改变. 在 RMIT 的“悬挂式酒吧”运 
动游戏项目 ${ }^{[54]}$ 中, 通过引人 SAR 数字元素, 以故 事为灵感，玩家可以在虚拟河流上尽可能长时间地 挂在练习杆上, 如图 10a 所示. BalanSAR 系统 ${ }^{[53,55]}$ 将交互式视频投影添加到传统的平衡锻炼和健身 登上，使这些锻炼更加有趣且富有挑战性.

\section{2 医疗辅助}

SAR 有着良好的环境感知和空间感知，使其 在许多以空间信息为关键因素的医疗场景得到了 应用. 传统的手术规划存在二维医学图像空间信 息有限等问题, 将 SAR 引入医疗辅助领域, 通过 数字化和三维技术, 将传统的二维图像信息投射 到实物人体模型或叠加到患者身体上, 使医生的 病患分析和手术治疗更加轻松精准. Wen 等 ${ }^{[56]}$ 提出 的投影相机系统，通过手术模型的投影、校正和配 准，直接在患者体内构建一个空间增强的真实手 术环境, 如图 10b 所示. 与传统的手术规划方法相 比, 这种增强的规划可以在实际病人身上进行三 维空间的手术规划, 并将规划集成到实时机器人 辅助的手术环境中. 实验结果表明, 该方法能促进 空间信息感知，并能在现场将虚拟规划传达给患 者. BodyExplorerAR 系统 ${ }^{[57]}$ 将图像和信息直接投 射到人体模型模拟器上, 可以提高学习者探索解 剖学、生理学和临床干预的能力. 例如, 用户可以 打开心脏的一个视图查看心电图, 然后服用各种 药物, 观察对心率和收缩力的影响. 随着技术的逐 步成熟, SAR 正在向检测诊断、治疗康复和病患管 理等应用环节渗透.

\section{3 教育娱乐}

由于 SAR 是基于物理空间的，在教育娱乐领 域中有重要的应用. 真实对象的投影映射内容可 以在数字空间中创建、编写、管理、交互和呈现，用 户有着手眼一致的可触交互体验. 此外, 低交互负 载和低认知负荷, 有助于锻炼学生快速适应这样 的学习环境.

近年来，博物馆也越来越多地将 SAR 应用于 故事讲述中, 通过在真实环境中讲述并体验故事, 可以更有效地给参观者留下深刻印象. 在早期的 一个案例研究 ${ }^{[58]}$ 中, 基于投影的光学透明显示器 被用来向博物馆参观者传达恐龙软组织重建的信 息, 将几层软组织覆盖在物理头骨上, 并对其功能 进行动画演示，该系统可以同时支持多个用户。在 这项工作用户研究的积极反馈表明, SAR 技术在教 育娱乐等领域的潜力.

在实验中, SAR 有助于培养学生的实际实验操 作技能. 现实空间中实验现象比纯虚拟仿真的实验
现象更具客观性, 数据也更具科学性. 近年来, 有 些研究者把 SAR 和教学联系起来, 取得了良好的效 果. Ukil 等 ${ }^{[59]}$ 基于 SAR 设计了带有基准标记的令牌, 用作交互的工具，来展示立方体、长方体和圆柱体 的属性, 激发了学生的兴趣. Laviole 等 ${ }^{[60]}$ 展示了交 互式投影教育应用一一色彩模型实验. 用户可以 通过移动物理标记组合颜色, 旨在让学生探索色 彩. 此外, 本研究团队开发了基于 SAR 的“认识磁 感线” 实验, 学生通过实物交互的形式移动三维打 印机打印出来的“磁铁”和“指南针”, 对象的纹理、 指南针的指向、磁感线均为投影出来的虚拟内容, 如图 10c 所示.

\section{4 艺 术}

利用 SAR 技术, 可以在视觉上改变和增强真 实世界物体的颜色、纹理或形状. 这种方法将物体 的视觉特性输人到投影仪中, 然后将其重新投影 到实物对象上, 以此呈现特定的视觉艺术效果. 因 为这些特点, SAR 在艺术、设计和舞台等得以广泛 应用. 如 HySAR 系统 ${ }^{\left[{ }^{[1]}\right.}$ 实现了 SAR 环境中高动态 范围和高分辨率的材质渲染. Zhang 等 ${ }^{[62]}$ 设计了一 种放置在舞台上的信号基站和演员携带的天线标 签, 以获得演员的位置, 呈现交互式的 SAR 舞台 效果. Gard 等 ${ }^{[63]}$ 在物体表面上通过投影仪投射新 的纹理控制动态移动对象的外观, 如图 $10 \mathrm{~d}$ 所示. Siegl 等 ${ }^{[64]}$ 使用深度传感器跟踪白色雕像后, 用多 台投影仪将形状一致且亮度最佳的图像实时投影 到雕像上.

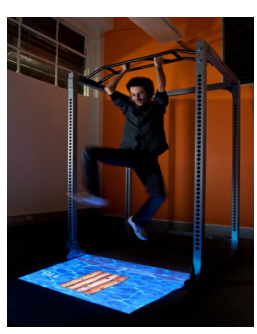

a. 悬挂式酒吧 ${ }^{[55]}$

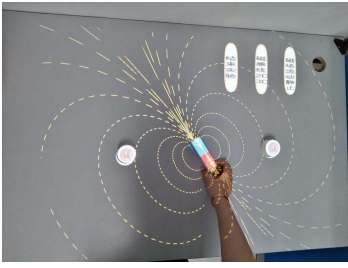

c. 磁感线 SAR 实验

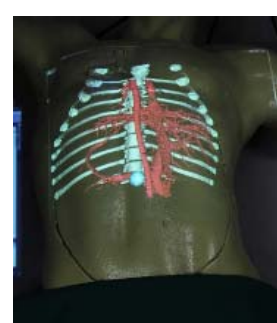

b. 手术规划 ${ }^{[57]}$

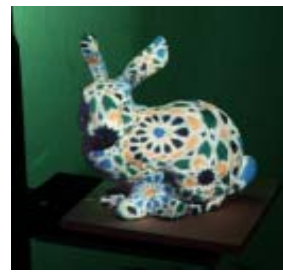

d. 投射纹理 ${ }^{[64]}$
图 10 SAR 应用场景

由以上分析可知, SAR 应用领域广泛，表 1 归 纳了上述应用系统. 
表 1 SAR 中典型应用系统

\begin{tabular}{llll}
\hline 应用场景 & \multicolumn{1}{c}{ 特点 } & \multicolumn{1}{c}{ 应用举例 } & \multicolumn{1}{c}{ 文献 } \\
\hline \multirow{4}{*}{ 运动锻炼 } & \multirow{3}{*}{ 利用技术增强物理 } & BalanSAR & {$[54]$} \\
& 空间促进行为改变 & Physical Education & {$[65]$} \\
& & 滑板运动 & {$[66]$} \\
\hline \multirow{2}{*}{ 医疗辅助 } & 在实物人体模型投 & 提供手术规划 & {$[55]$} \\
& 影视图 & BodyExplorerAR & {$[56]$} \\
\hline \multirow{3}{*}{ 教育娱乐 } & \multirow{2}{*}{ 手眼一致的可触交 } & 博物馆展示 & {$[58]$} \\
& 互效果 & 交互式教学体验 & {$[59]$} \\
& & 实验应用系统 & {$[60]$} \\
\hline \multirow{4}{*}{ 艺术 } & \multirow{4}{*}{} & HySAR & {$[61]$} \\
& 增强呈现特定的视 & 舞台效果 & {$[62]$} \\
& 觉艺术效果 & 改变对象外观 & {$[63]$} \\
& & 雕像上色 & {$[33-34,64]$} \\
\hline
\end{tabular}

\section{5 未来发展趋势}

SAR 技术为探索物理和虚拟世界之间的交互 新方法创造了机会, 越来越多的信号可被捕捉作 为交互的输人. 未来, 要使 SAR 系统变得高效、鲁 棒且易用，需要在多方面取得突破与发展.

\section{1 交互方式更自然}

为了使用户更专注于交互内容本身，而不被 交互设备所干扰, 需要在显式人机交互的基础上 融人隐式交互模式，脑机交互、眼动和情感等隐式 交互方式是未来人机交互的重要发展方向. 此外, 显式交互模式中 TUI 将数字信息耦合到日常物理 对象和环境上来扩展真实的物理世界, 这种研究 利用自然的方式将物理世界本身变成一个交互界 面. 实物交互采用基于虚拟化身的交互隐喻，强调 将物理实体映射到计算机的数字空间, 即直接将人 们在现实生活中与物体的交互动作映射为和计算机 虚拟空间交互过程的用户界面，符合人机交互界面 设计易学习和自然 2 个方面的要求 ${ }^{[67]}$. 提高了用户 和信息之间交互的易读性和无缝性，无交互负载， 交互意图易判断, 输人输出空间不隔离, 可多用户 协同操作, 这也将是未来 SAR 的重要交互手段.

\section{2 交互遮挡处理方法更智能}

在交互技术方面, 需要提高虚拟物体在真实 环境中的位置和运动跟踪的精准度, 提高 SAR 系 统在不同交互场景中的效率、准确度和鲁棒性, 神 经网络可能是未来解决遮挡问题的重要思路. 针 对 SAR 环境中的遮挡处理, 传感硬件方面的改进 无疑可以获得更高的分辨率和场景范围，但这些 改进可能会达到上限. 当使用神经网络时, 可以在 场景中挑出视觉线索来估计三维结构, 就像人类 估计距离的方式一样. 可以在大型图像数据集上
进行训练, 分割出场景中的物体, 然后识别它们以 估计深度, 从而正确地处理物体之间的遮挡关系.

\section{3 系统的使用场景更广泛}

在使用场景受限方面, 要降低系统的配置难 度, 提高系统的便携性和可移动性. 传统的 SAR 系统校准方法通常需要相机和投影仪之间的对应 关系估计校准信息. 但是，构建投影仪相机系统需 要正确安装和配置相机, 以便清唽地观察投影图 案, 并且在校准过程中相机必须是固定的. 在某些 情况下, 要满足这些要求可能很困难, 并且仅将摄 像机用于系统部署是并不经济的. 这种系统往往 不能由普通用户部署到应用场景中, 从而限制了 其使用. 近年来, 已经有研究者提出使用移动相机 和空间编码的投影仪校准方法. 可以允许用户手 持摄像机(如智能手机的内置相机)进行投影仪的 校准, 减少摄像机设置所需工作量和时间 ${ }^{[68]}$. 但 该校准过程需要提取特征, 依然会受到光照或噪 声等因素的影响. 因此, 未来需要研究设备简易、 并解决光照等因素影响的校准方法, 从而提升系 统的易用性. 此外, 微型投影仪将会给 SAR 中应 用系统的便携性带来巨大的变革, 最近, 很多公司 推出了手持式微型投影仪. 例如，百度的“DuRing” 戒指投影仪的外观和一枚戒指相当, 可直接佩戴 在手指上, 可投影出 $1280 \times 800$ 像素的分辨率. 未 来的微型投影仪可以嵌人或连接到手机、掌上电脑 (personal digital assistant, PDA)或相机中. 此外, 当用户需要呈现隐私内容时, 可以用闪光灯等形 式提醒用户保护好自己的内容不被其他人偷窥, 同时提醒其他人此时不要偷窥用户的呈现内容.

\section{4 与社会、文化和心理现象研究相结合}

研究 SAR 背后的社会、文化和心理现象, 将 有助于 SAR 产品的开发. 在交互数字媒体的背景 下，捕获、表示和处理有关真实和虚拟空间的人类 情感和认知行为是人机交互的基本问题. 随着人 机界面和数据捕获技术的进步, SAR 可以更好地呈 现知识、经验和情感内容, 但目前针对心理和情感 用户界面的研究还较少.

\section{5 新型的成像方式}

水幕、气幕等特殊介质显示, 以及光场显示和 无介质空中悬浮成像等新型成像方式的产生与发 展, 也将是未来 SAR 的重要发展方向. 与物理空 间结合更紧密、介质越来越隐蔽、显示方式更贴近 人的日常生活等, 都是新型成像方式追求的重要 目标. 新的成像方式不仅带来了新的信息显示与 接收方式，也必将带来新的人机交互方式. 


\section{6 结 语}

SAR 经过多年的发展，相关核心技术取得了 显著的进步, 展示出蓬勃的发展趋势, 人机交互是 SAR 的重要支撑技术，也是近年来国内外的研究 热点. 在交互对象的虚实融合技术的基础上，基于 触控的交互、基于手势或姿势的交互、基于控制器 的交互、手持投影仪交互、实物交互、多模态交互 等技术的广泛应用，极大地拓展了 SAR 中的交互 通道, 为实现 SAR 中自然、高效的人机交互做出 了巨大贡献. 然而，由于现有软硬件技术上存在着 一定的局限和制约，目前的交互技术仍在稳定性、 准确性和实时性上存在缺陷, 今后仍需开发更多 的技术来支撑 SAR 交互的发展。

\section{参考文献(References):}

[1] Raskar R, Welch G, Fuchs H. Spatially augmented reality[C] //Proceedings of the International Workshop on Augmented Reality: Placing Artificial Objects in Real Scenes. Boca Raton: AK Peters/CRC Press, 1999: 63-72

[2] Mine M R, van Baar J, Grundhofer A, et al. Projection-based augmented reality in Disney theme parks[J]. Computer, 2012, 45(7): 32-40

[3] Raskar R, Welch G, Low K L, et al. Shader lamps: animating real objects with image-based illumination[C] //Proceedings of Eurographics Workshop on Rendering Techniques. Aire-la-Ville: Eurographics Association Press, 2001: 89-102

[4] Raskar R, Welch G, Cutts M, et al. The office of the future: a unified approach to image-based modeling and spatially immersive displays[C] //Proceedings of the 25th Annual Conference on Computer Graphics and Interactive Techniques. New York: ACM Press, 1998: 179-188

[5] Bimber O, Raskar R. Spatial augmented reality: merging real and virtual worlds[M]. Boca Raton: AK Peters/CRC Press, 2005: 71-90

[6] Bao Hujun, Zhang Guofeng, Qin Xueying, et al. Augmented reality: principles, algorithms and applications[M]. Beijing: Science Press, 2019: 220-245(in Chinese)

(鲍虎军, 章国锋, 秦学英, 等. 增强现实：原理、算法与应 用[M]. 北京: 科学出版社, 2019: 220-245)

[7] Piper B, Ratti C, Ishii H. Illuminating clay: a 3D tangible interface for landscape analysis[C] //Proceedings of the SIGCHI Conference on Human Factors in Computing Systems. New York: ACM Press, 2002: 355-362

[8] Wilson A D. Depth-sensing video cameras for 3D tangible tabletop interaction[C] //Proceedings of the IEEE International Workshop on Horizontal Interactive Human-Computer Systems. Los Alamitos: IEEE Computer Society Press, 2007: 201-204

[9] Bimber O, Frohlich B, Schmalsteig D, et al. The virtual showcase[J]. IEEE Computer Graphics and Applications, 2001,
21(6): 48-55

[10] Li N, Sharlin E, Sousa M C. Duopography: using back-of-device multi-touch input to manipulate spatial data on mobile tangible interactive topography[C] //Proceedings of the SIGGRAPH Asia 2017 Mobile Graphics \& Interactive Applications. New York: ACM Press, 2017: Article No.20

[11] Harrison C, Benko H, Wilson A D. OmniTouch: wearable multitouch interaction everywhere[C] //Proceedings of the 24th annual ACM Symposium on User Interface Software and Technology. New York: ACM Press, 2011: 441-450

[12] Hochreiter J, Daher S, Nagendran A, et al. Optical touch sensing on nonparametric rear-projection surfaces for interactive physical-virtual experiences[J]. Presence: Teleoperators and Virtual Environments, 2016, 25(1): 33-46

[13] Dung L R, Lai G Y, Wu Y Y. Shadow touching for interactive projectors[C] //Proceedings of the IEEE International Conference on Acoustics, Speech and Signal Processing. Los Alamitos: IEEE Computer Society Press, 2013: 1798-1802

[14] Wilson A D, Benko H. Combining multiple depth cameras and projectors for interactions on, above and between surfaces[C] //Proceedings of ACM Symposium on User Interface Software and Technology. New York: ACM Press, 2010: 273-282

[15] Chen Rui, Li Hui, Hou Yibin, et al. Multi-direction projecting interactive system driven by facial orientation[J]. Journal of Chinese Computer Systems, 2007, 28(4): 706-709(in Chinese) (陈锐，李辉，侯义斌，等. 由人脸朝向驱动的多方向投影交 互系统[J]. 小型微型计算机系统, 2007, 28(4): 706-709)

[16] Kumar S P, Pandithurai O. Sixth sense technology[C] //Proceedings of the International Conference on Information Communication and Embedded Systems. Los Alamitos: IEEE Computer Society Press, 2013: 947-953

[17] Sun Chao, Zhang Mingmin, Li Yang, et al. Hand based natural interaction in augmented reality[J]. Journal of Computer-Aided Design \& Computer Graphics, 2011, 23(4): 697-704(in Chinese)

(孙超, 张明敏, 李扬, 等. 增强现实环境下的人手自然交互 [J]. 计算机辅助设计与图形学学报, 2011, 23(4): 697-704)

[18] Bandyopadhyay D, Raskar R, Fuchs H. Dynamic shader lamps: painting on movable objects[C] //Proceedings of the IEEE and ACM International Symposium on Augmented Reality. Los Alamitos: IEEE Computer Society Press, 2001: 207-216

[19] Chen Feng, Liu Yue, Wang Yongtian. Real-time drawing technique for textured surface based on multi-projector system[J]. Journal of Beijing University of Aeronautics and Astronautics, 2015, 41(3): 466-471(in Chinese)

(陈锋, 刘越, 王涌天. 基于多通道投影系统的纹理表面实 时绘制技术 [J]. 北京航空航天大学学报，2015，41(3): 466-471)

[20] Roo J S, Hachet M. Towards a hybrid space combining spatial augmented reality and virtual reality[C] //Proceedings of the IEEE Symposium on 3D User Interfaces(3DUI). Los Alamitos: IEEE Computer Society Press, 2017: 195-198

[21] Cao X, Forlines C, Balakrishnan R. Multi-user interaction using handheld projectors[C] //Proceedings of the 20th Annual ACM Symposium on User Interface Software and Technology. New York: ACM Press, 2007: 43-52

[22] Cao X, Balakrishnan R. Interacting with dynamically defined 
information spaces using a handheld projector and a pen[C] //Proceedings of the 19th Annual ACM Symposium on User Interface Software and Technology. New York: ACM Press, 2006: 225-234

[23] Santos R, Correia N. Haunted house: an interactive experience using a pico projector[C] //Proceedings of the 12th International Conference on Advances in Computer Entertainment Technology. New York: ACM Press. 2015: 1-4

[24] Willis K D D, Poupyrev I, Hudson S E, et al. SideBySide: ad-hoc multi-user interaction with handheld projectors[C] //Proceedings of the 24th Annual ACM Symposium on User Interface Software and Technology. New York: ACM Press, 2011: 431-440

[25] Ullmer B, Ishii H. The metaDESK: models and prototypes for tangible user interfaces[C] //Proceedings of the 10th annual ACM Symposium on User Interface Software and Technology. New York: ACM Press, 1997: 223-232

[26] Jones C E, Maquil V. Twist, shift, or stack? Usability analysis of geospatial interactions on a tangible tabletop[C] //Proceedings of the 1st International Conference on Geographical Information Systems Theory, Applications and Management. Los Alamitos: IEEE Computer Society Press, 2015: 1-8

[27] de Raffaele C, Smith S, Gemikonakli O. Teaching and learning queueing theory concepts using tangible user interfaces[C] //Proceedings of the IEEE International Conference on Teaching, Assessment, and Learning for Engineering. Los Alamitos: IEEE Computer Society Press, 2016: 194-201

[28] Attard G, de Raffaele C, Smith S. TangiBoard: a toolkit to reduce the implementation burden of tangible user interfaces in education[C] //Proceedings of the 13th IEEE International Conference on Application of Information and Communication Technologies. Los Alamitos: IEEE Computer Society Press, 2019: $1-7$

[29] de Raffaele C, Buhagiar G, Smith S, et al. Designing a table-top tangible user interface system for higher education[C] //Proceedings of the International Conference on Smart Systems and Technologies. Los Alamitos: IEEE Computer Society Press, 2017: 285-291

[30] Sano M, Matsumoto K, Thomas B H, et al. Rubix: dynamic spatial augmented reality by extraction of plane regions with a RGB-D camera[C] //Proceedings of the IEEE International Symposium on Mixed and Augmented Reality. Los Alamitos: IEEE Computer Society Press, 2015: 148-151

[31] Gervais R, Roo J S, Hachet M. Tangible viewports: getting out of flatland in desktop environments[C] //Proceedings of the TEI'16: 10th International Conference on Tangible, Embedded, and Embodied Interaction. New York: ACM Press, 2016: 176-184

[32] Oswald P, Tost J, Wettach R. The real augmented reality: real-time game editor in a spatial augmented environment[C] //Proceedings of the 11th Conference on Advances in Computer Entertainment Technology. New York: ACM Press, 2014: Article No.32

[33] Zhou Y, Xiao S J, Tang N, et al. Pmomo: projection mapping on movable 3D object[C] //Proceedings of the CHI Conference on Human Factors in Computing Systems. New York: ACM
Press, 2016: 781-790

[34] Kurth P, Lange V, Siegl C, et al. Auto-calibration for dynamic multi-projection mapping on arbitrary surfaces[J]. IEEE Transactions on Visualization and Computer Graphics, 2018, 24(11): 2886-2894

[35] Kagami S, Hashimoto K. Animated stickies: fast video projection mapping onto a markerless plane through a direct closed-loop alignment[J]. IEEE Transactions on Visualization and Computer Graphics, 2019, 25(11): 3094-3104

[36] Punpongsanon P, Iwai D, Sato K. FleXeen: visually manipulating perceived fabric bending stiffness in spatial augmented reality[J]. IEEE Transactions on Visualization and Computer Graphics, 2020, 26(2): 1433-1439

[37] Sueishi T, Oku H, Ishikawa M. Robust high-speed tracking against illumination changes for dynamic projection mapping[C] //Proceedings of the IEEE Virtual Reality. Los Alamitos: IEEE Computer Society Press, 2015: 97-104

[38] Huang Jin, Han Dongqi, Chen Yineng, et al. A survey on human-computer interaction in mixed reality[J]. Journal of Computer-Aided Design \& Computer Graphics, 2016, 28(6): 869-880(in Chinese)

(黄进，韩冬奇，陈毅能，等. 混合现实中的人机交互综述 [J]. 计算机辅助设计与图形学学报, 2016, 28(6): 869-880)

[39] Benko H, Wilson A D, Zannier F. Dyadic projected spatial augmented reality[C] //Proceedings of the 27th Annual ACM Symposium on User Interface Software and Technology. New York: ACM Press, 2014: 645-655

[40] Mistry P, Maes P, Chang L. WUW - wear Ur world: a wearable gestural interface[C] //Proceedings of the CHI'09 Extended Abstracts on Human Factors in Computing Systems. New York: ACM Press, 2009: 4111-4116

[41] Oviatt S L. Mutual disambiguation of recognition errors in a multimodel architecture[C] //Proceedings of the SIGCHI conference on Human Factors in Computing Systems. New York: ACM Press, 1999: 576-583

[42] Luo T R, Zhang M M, Pan Z G, et al. Dream-Experiment: a MR user interface with natural multi-channel interaction for virtual experiments[J]. IEEE Transactions on Visualization and Computer Graphics, 2020, 26(12): 3524-3534

[43] Liu Yiran, Yang Xubo. Camera-based multi-projector radiometric compensation system[J]. Journal of Image and Graphics, 2011,16(3): 342-349(in Chinese)

(刘一然, 杨旭波. 基于相机的多投影光度补偿系统 [J]. 中 国图象图形学报, 2011, 16(3): 342-349)

[44] Raskar R, Brown M S, Yang R G, et al. Multi-projector displays using camera-based registration[C] //Proceedings of the Proceedings Visualization'99: Celebrating Ten Years. Los Alamitos: IEEE Computer Society Press, 1999: 161-168

[45] Rehg J M, Flagg M, Cham T J, et al. Projected light displays using visual feedback[C] //Proceedings of the 7th International Conference on Control, Automation, Robotics and Vision. Los Alamitos: IEEE Computer Society Press, 2003: 926-932

[46] Yang H X, Shao L, Zheng F, et al. Recent advances and trends in visual tracking: a review[J]. Neurocomputing, 2011, 74(18): 3823-3831

[47] Liu Yue, Wang Yongtian, Yan Dayuan. Research on display technology of augmented reality system[C]//Proceedings of the 
3rd International Conference on Frontiers in Optical Imaging Technology and Application. Beijing: Chinese Society for Optical Engineering Press, 2004: 19-33(in Chinese)

(刘越, 王涌天，间达远. 增强现实系统显示技术研究[C] // 第三届全国数字成像技术及相关材料发展与应用学术研讨 会论文摘要集. 北京: 中国光学工程学会出版社, 2004: 19-33)

[48] Rolland J P, Baillot Y, Goon A A. A survey of tracking technology for virtual environments[J]. Fundamentals of Wearable Computers and Augmented Reality, 2001, 1(1): 67-112

[49] Lowe D G. Object recognition from local scale-invariant features[C] //Proceedings of the 7th IEEE International Conference on Computer Vision. Los Alamitos: IEEE Computer Society Press, 1999: 1150-1157

[50] Cortes G, Marchand E, Brincin G, et al. MoSART: mobile spatial augmented reality for 3D interaction with tangible objects[J]. Frontiers in Robotics and AI, 2018, 5(93): 1-13

[51] Xu Weipeng, Weng Dongdong, Liu Yue, et al. Depth camera based dynamic projector calibration for spatial augmented reality[J]. Journal of System Simulation, 2013, 25(9): 2097-2103 (in Chinese)

(徐维鹏, 翁冬冬, 刘越, 等. 基于深度相机的空间增强现实 动态投影标定 [J]. 系统仿真学报, 2013, 25(9): 2097-2103)

[52] Bimber O, Frohlich B. Occlusion shadows: using projected light to generate realistic occlusion effects for view-dependent optical see-through displays[C] //Proceedings of International Symposium on Mixed and Augmented Reality. Los Alamitos: IEEE Computer Society Press, 2002: 186-319

[53] Mast D, Bosman M, Schipper S, et al. Adding interactivity to BalanSAR: a spatial augmented reality game for balancing in physical education[C] //Proceedings of the Extended Abstracts Publication of the Annual Symposium on Computer-Human Interaction in Play. New York: ACM Press, 2017: 383-389

[54] Mueller F, Toprak C, Graether E, et al. Hanging off a bar[C] //Proceedings of the CHI'12 Extended Abstracts on Human Factors in Computing Systems. New York: ACM Press, 2012: 1055-1058

[55] Mast D, Bosman M, Schipper S, et al. BalanSAR: using spatial augmented reality to train children's balancing skills in physical education[C] //Proceedings of the 11th International Conference on Tangible, Embedded, and Embodied Interaction. New York: ACM Press, 2017: 625-631

[56] Wen R, Nguyen B P, Chng C B, et al. In situ spatial AR surgical planning using projector-Kinect system[C] //Proceedings of the 4th Symposium on Information and Communication Technology. New York: ACM Press, 2013: 164-171

[57] Samosky J T, Nelson D A, Wang B, et al. BodyExplorerAR: enhancing a mannequin medical simulator with sensing and projective augmented reality for exploring dynamic anatomy and physiology[C] //Proceedings of the 6th International Conference on Tangible, Embedded and Embodied Interaction. New York: ACM Press, 2012: 263-270

[58] Bimber O, Gatesy S M, Witmer L M, et al. Merging fossil specimens with computer-generated information[J]. Computer, 2002, 35(9): 25-30

[59] Ukil M, Sorathia K. Re-defining learning experience for kids[C] //Proceedings of the Students Technology Symposium. Los Alamitos: IEEE Computer Society Press, 2014: 218-223

[60] Laviole J, Thevin L, Albouys-Perrois J, et al. Nectar: multi-user spatial augmented reality for everyone: three live demonstrations of educative applications[C] //Proceedings of the Virtual Reality International Conference Laval Virtual. New York: ACM Press, 2018: Article No.27

[61] Hiroi Y, Itot Y, Hamasaki T, et al. HySAR: hybrid material rendering by an optical see-through head-mounted display with spatial augmented reality projection[C] //Proceedings of the IEEE Virtual Reality. Los Alamitos: IEEE Computer Society Press, 2017: 211-212

[62] Zhang Y X, Shen Y R, Zhang W W, et al. Design of an interactive spatial augmented reality system for stage performance based on UWB positioning and wireless triggering technology[J]. Applied Sciences, 2019, 9(7): 1318

[63] Gard N, Hilsmann A, Eisert P. Projection distortion-based object tracking in shader lamp scenarios[J]. IEEE Transactions on Visualization and Computer Graphics, 2019, 25(11): 3105-3113

[64] Siegl C, Colaianni M, Thies L, et al. Real-time pixel luminance optimization for dynamic multi-projection mapping[J]. ACM Transactions on Graphics, 2015, 34(6): Article No.237

[65] Mast D, de Krom J, de Vries S. Exploring the application of interactive video projection in physical education[C] //Proceedings of the 9th International Conference on Tangible, Embedded, and Embodied Interaction. New York: ACM Press, 2015: 551-555

[66] Pijnappel S, Mueller F. Copy paste skate[C] //Proceedings of the CHI'13 Extended Abstracts on Human Factors in Computing Systems. New York: ACM Press, 2013: 2863-2864

[67] Huang Chaodian, Chen Hui, Peng Xiaolan, et al. Tangible user interface for speech rehabilitation training[J]. Journal of Computer-Aided Design \& Computer Graphics, 2016, 28(9): 1560-1570(in Chinese)

(黄朝殿, 陈辉, 彭晓兰, 等. 实物交互在言语康复训练中的 应用 $[J]$. 计算机辅助设计与图形学学报，2016，28(9): 1560-1570)

[68] Xie C, Shishido H, Kameda Y, et al. A projector calibration method using a mobile camera for projection mapping system[C] //Proceedings of the IEEE International Symposium on Mixed and Augmented Reality Adjunct. Los Alamitos: IEEE Computer Society Press, 2019: 261-262 